\title{
COMERCIO EXTERIOR Y DESARROLLO ECONOMICO: REFLEXIONES SOBRE EL CASO ANDALUZ EN LA SEGUNDA MITAD DEL SIGLO XIX '
}

\author{
CLARA EUGENIA NUÑEZ \\ New York University
}

\section{Introducción}

Se ha escrito mucho sobre las causas del atraso andaluz, sin que hasta el momento exista ninguna explicación definitiva. La mayor parte de los planteamientos que se han hecho, unos mejor fundamentados que otros, son insatisfactorios por su monocausalidad. En términos generales, se busca al gran culpable del atraso relativo del Sur con respecto a otras regiones peninsulares, atraso que no se inició hasta bien entrada la segunda mitad del siglo xIx, al invertirse la tendencia expansionista seguida hasta entonces.

Entre los argumentos más conocidos destaca, en primer lugar, el que hace referencia al sistema de propiedad de la tierra. La consolidación del latifundio gracias a las sucesivas desamortizaciones, se ha dicho, impidió que tuviera lugar una verdadera revolución agrícola en el campo andaluz. La descapitalización de los grandes propietarios, junto con el crecimiento desmesurado del número de los jornaleros sin tierras, es decir, de la disponibilidad de mano de obra barata, actuaron de freno a la introducción de mejoras técnicas. De ahí que se mantuviera un nivel de productividad muy bajo y se acentuaran las desigualdades en la distribución de la renta. También se ha considerado la aparición de «enclaves extranjeros», especialmente en la minería, responsable del escaso impacto de la explotación de esta riqueza natural sobre la economía andaluza. Esta tesis hace hincapié en la dependencia de Andalucía con respecto a algunos de los países más adelantados del norte de Europa. Asimismo, se ha argumentado que la falta de una fuerte mentalidad empresarial impidió a Andalucía aprovechar al máximo sus propias ventajas comparativas en relación con otras regiones peor dotadas de recursos naturales. Finalmente, se ha llamado la atención sobre la existencia de ciertas barreras institucionales tales

' Este trabajo está basado en mi Memoria de Licenciatura, dirigida por Manuel Titos Leandro Prados y a Nicolás Sánchez-Albornoz (que sugirió el tema y dirigió mi trabajo en la Universidad de Nueva York) quiero manifestarles mi agradecimiento, aunque no atribuirles los defectos del trabajo. Algunas de las ideas aquí expuestas las desarrollaré en mi tesis doctoral. 
como unos aranceles proteccionistas muy elevados y la concesión del monopolio de emisión al Banco de España, medidas que habrían perjudicado, respectivamente, al comercio de exportación andaluz y a su sistema bancario ${ }^{2}$.

Si bien estas interpretaciones tienen alguna justificación, falta aún un estudio que enmarque a los distintos "responsables» del atraso andaluz en el contexto adecuado y determine la importancia relativa de cada uno de ellos. Este estudio debería prestar atención no sólo a los sectores atrasados, sino también a los que resultaron más dinámicos en un momento u otro. Para algunos de estos casos existen ya buenas monografías, como para la Banca andaluza, algunos distritos mineros, ciertos núcleos de agricultura especializada y los transportes, en concreto la construcción del ferrocarril. Todas estas actividades se encuentran estrechamente vinculadas a otro sector dinámico, el comercio exterior, cuyo estudio, sin embargo, está aún por hacer y sobre el que propongo aquí unas reflexiones ${ }^{3}$.

Entre otras razones que havan podido mantener a los historiadores alejados de este tema, destacan el problema formal de las fuentes y el problema teórico de explicar las conexiones entre el comercio exterior y el desarrollo económico. El primer obstáculo lo he resuelto, provisionalmente, utilizando la Estadistica General del Comercio Exterior de España, tan sólo para productos típicamente andaluces, como los vinos de Jerez, el esparto o el plomo y el cobre, y con la ayuda ocasional de los Informes Consulares Británicos ${ }^{4}$.

La dificultad teórica es mayor, si cabe. El comercio exterior no es una condición indispensable o necesaria, ni tampoco suficiente, para explicar el crecimiento económico o su ausencia. Sí puede ser, sin embargo, un factor acelerador, desacelerador o de equilibrio en el proceso de cambio y crecimiento económico. El que actúe de una u otra forma depende, en gran parte, de su relación con el resto de la economía, es decir, de que su expansión esté dirigida por la demanda o esté basada en un mecanismo autónomo por parte de la oferta. Es, pues, una vía de desarrollo que no ha resultado igualmente satisfactoria para todas las regiones que han optado por ella. El problema reside, así, en determinar si el comercio exterior es un «motor de transmisión

2 Sobre estos temas véanse, por ejemplo, Bernal (1974 y 1979), Bernal y Drain (1975), Cruz (1980), Nadal (1972 y 1975), García Montoro (1978), Castejón Montijano (1977), Jiménez Quintero (1979), Titos Martínez et at. (1980) y Tedde (1981). Este último incluye una bibliografía comentada y más completa de estos temas.

3 Algunos de los títulos más notables son: Sánchez-Albornoz (1968), Tortella (1973), Tedde (1981), Titos Martínez (1978 y 1980), Castejón Montijano (1979), Avery (1974), Harvey (1981), Checkland (1967), Nadal (1972 y 1975), Muñoz Dueñas (1979), Morilla (1978 y 1984) y Tedde (1978 y 1980). Para una bibliografía más amplia véase también Tedde (1981).

4 Para la cuestión de la validez de las estadísticas, véanse V. A. Alvarez (1945), G. Tortella, P. Martín Aceña, J. Sanz y S. Zapata (1978) y L. Prados de la Escosura (1981). En este trabajo he utilizado series tomadas de la EGCE y algunos datos de los CR. 
del crecimiento», como llegó a afirmar Nurkse, o si, por el contrario, no hay suficiente evidencia histórica que permita relacionar unas condiciones favorables de la demanda externa con un proceso de desarrollo económico regional, como mantiene Kravis ${ }^{5}$.

Según Nurkse, el crecimiento que experimentaron las economías del centro, es decir, la Europa septentrional, con Gran Bretaña a la cabeza, actuó como un poderoso elemento de arrastre sobre las economías periféricas, entre las que cabe incluir en un primer momento a la Europa mediterránea y oriental $^{6}$. La industrialización de los países del centro generó una fuerte y creciente demanda de materias primas y productos alimenticios, demanda a la que las regiones periféricas respondieron con la especialización en su producción. La demanda externa permitió, pues, el aprovechamiento de los recursos naturales de cada región y fue la base de su posterior crecimiento económico. Aunque Nurkse se refería específicamente a las «zonas de poblamiento reciente», la idea del comercio exterior como motor de transmisión se generalizó más ampliamente.

Kravis partió de un supuesto implícito en la teoría de Nurkse para cuestionar su validez. Esto es, si el comercio exterior es un motor de transmisión, el atraso económico podría explicarse por el aislamiento de diversas regiones y por su falta de comunicación con el centro. $Y$ esto no es así en los países tropicales durante la segunda mitad del siglo XIX, como puso de manifiesto el propio Kravis en sus estudios. Este autor sugiere, como explicación alternativa, que el efecto más importante del comercio exterior para una región en crecimiento es el de permitirle encontrar y fomentar aquellas actividades para las que tuviera ventajas comparativas. El atraso económico de una región no se explica, pues, por la falta de comercio exterior, sino por las condiciones internas que determinan que su impacto sea mayor o menor. De ahí que sean estas condiciones las que voy a intentar encontrar para explicar el limitado impacto del comercio exterior de Andalucía sobre la economía regional.

\section{Características del comercio exterior andaluz}

A lo largo del siglo xix destaca un aumento considerable de la participación andaluza en el sector exterior español. Antes de la independencia de las colonias del continente americano, Andalucía apenas contribuía en un 10 por 100 al comercio exterior de España. La situación cambió notablemente desde finales de los años veinte hasta comienzos de los ochenta. Durante este pe-

' Kindleberger (1968), Nurkse (1961) y Kravis (1970).

- Para el concepto de periferia aplicado a los países mediterráneos, véase I. T. Berend y G. Ranki (1980). 
ríodo, el Mediodía peninsular alcanzó a representar casi la mitad del comercio exterior. En este cambio fue decisiva la reorganización y reestructuración del comercio exterior español tras la pérdida de los mercados coloniales y el consiguiente aumento de las exportaciones de materias primas y productos agrícolas a los países de la Europa del Norte. Durante las décadas centrales del siglo xIx, el dinamismo del sector exterior andaluz fue superior al del comercio exterior español. Finalmente, entre 1880, aproximadamente, y 1913, el comercio andaluz supuso entre un cuarto y un quinto del comercio español y, por tanto, refleja un descenso en relación con el período anterior que no pudo compensar el aumento de las exportaciones de minerales ${ }^{7}$.

Uno de los principales mercados para los productos andaluces fue, durante todo el período, Gran Bretaña. En este aspecto, pues, Andalucía no se apartaba de las pautas dominantes del comercio exterior español. Ahora bien, la vinculación entre las economías británica y andaluza parece más estrecha aún que la existente entre las Islas y la Península en su conjunto. La compenetración alcanzada entre ambos mercados se manifiesta en el paralelismo existente entre la evolución de la economía británica y las exportaciones andaluzas durante la pasada centuria: hay una primera etapa de expansión y crecimiento acelerado que se termina en torno a 1873 , fecha a partir de la cual la tendencia se interrumpe y el crecimiento prosigue a ritmo inferior.

La composición de las exportaciones andaluzas es también típica del comercio exterior español, es decir, dominan en ella las materias primas y los productos agrícolas. El Mediodía contaba con ventajas indudables sobre otras regiones españolas a la hora de hacer frente a este tipo de demanda. Por una parte, Andalucía tiene importantes recursos naturales, tales como yacimientos de minerales escasos en el resto de Europa, y, por su situación geográfica, reúne las condiciones necesarias para desarrollar una agricultura muy especializada, como la dedicada a la producción de vinos, pasas, aceite, etc. Por otra parte, sus recursos se encuentran óptimamente situados con vistas a la exportación, por su relativa proximidad al mar y por su no menos importante cercanía a los mercados del norte de Europa.

Estos rasgos generales se confirman al desglosar el comercio de exportación por productos. En el cuadro 1 se establecen los porcentajes de participación de ciertos productos típicamente andaluces en el total del comercio exterior español para los años centrales del siglo. Aun contando con numerosas ausencias, algunas tan notables como el aceite de oliva o las pasas, queda de manifiesto ta importancia de los productos andaluces dentro del sector exterior español. Grosso modo podría afirmarse que estos productos representaban entre un 30 y un 50 por 100 de las exportaciones andaluzas a mediados

\footnotetext{
${ }^{7}$ L. Prados de la Escosura (1982) y una conferencia inédita en Sevilla, septiembre 1983.
} 
del siglo xix. Veamos con algún detalle la evolución de las exportaciones en algunos de estos productos.

\section{CUADRO 1}

Exportación de algunos productos andaluces. Porcentaje respecto al valor de las exportaciones totales españolas. Medias quinquenales

\begin{tabular}{|c|c|c|c|c|c|c|}
\hline & $A \tilde{n} o s$ & $\begin{array}{c}(1) \\
\text { Plomo } \\
\text { en barras }\end{array}$ & $\begin{array}{c}(2) \\
\text { Mineral } \\
\text { de cobre }\end{array}$ & $\begin{array}{c}(3) \\
\text { Vino } \\
\text { de Jerez }\end{array}$ & $\begin{array}{c}\text { (4) } \\
\text { Esparto }\end{array}$ & $\begin{array}{l}\text { (5) } \\
\text { Total }\end{array}$ \\
\hline $\begin{array}{l}1860-64 \\
1865-69 \\
1870-74 \\
1875-79 \\
1880-84\end{array}$ & 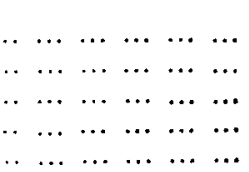 & $\begin{array}{l}6 \\
5,6 \\
5,3 \\
5,2 \\
4,2\end{array}$ & $\frac{-}{7}$ & $\begin{array}{r}13,9 \\
13,4 \\
20 \\
11,7 \\
7,5\end{array}$ & $\begin{array}{l}0,5 \\
2 \\
1,5 \\
1,2 \\
-\end{array}$ & $\begin{array}{l}20,4 \\
21 \\
26,8 \\
25,1 \\
17,9\end{array}$ \\
\hline
\end{tabular}

Fuentes: Estadística General del Comercio Exterior de España, Informes Consulares Británicos (precios del esparto) y Nadal (1975) (apéndice sobre la producción de plomo en España).

La exportación de sherry - denominación anglizada bajo la que se conoce un tipo de vino de Jerez en el extranjerc- es un fenómeno anterior al siglo xIx. Dado el escaso consumo interno de este caldo - los españoles no habían desarrollado el gusto por este particular tipo de vinos, ni gozaban de una renta que les permitiera su consumo en gran escala-, no sorprende que su producción estuviera orientada al mercado exterior, y en especial al británico, que consumía aproximadamente un 90 por 100 a comienzos de siglo ${ }^{8}$. Ahora bien, en los años cincuenta la demanda británica de sherry superó la capacidad de producción de Jerez. La oferta, por naturaleza inelástica, dada la escasez de tierras adecuadas al cultivo de esta viña, y sujeta, asimismo, a frecuentes cambios coyunturales, se vio disminuida por una serie de malas cosechas en los años cincuenta y la plaga del oidium en los primeros años sesenta ${ }^{9}$. La disminución de la oferta, tanto en términos absolutos como en términos relativos, determinó un fuerte aumento en la cotización del sherry. Su precio medio en 1864 fue de $36 £$ por bota, lo que supuso un aumento de un 70 por 100 para el período comprendido entre 1855 y $1864^{10}$. El aumento de la demanda de sherry, unido al salto en su cotización, dieron lugar a una

' F. Quiros (1962), p. 29.

- CR 1866, vol. LXX, p. 328.

10 CR 1865, vol. LIII, p. 659. 
expansión de los viñedos sobre tierras poco adecuadas, tanto en la zona de Jerez propiamente dicha como en regiones adyacentes de las provincias de Huelva, Sevilla e incluso Córdoba ". Los vinos que se obtuvieron no podían competir ni en cuerpo ni en aroma con el sherry, pese a lo cual se exportaron a Gran Bretaña como si de sherry se tratara. Se cubrió, pues, de esta forma la creciente demanda británica, pero a costa de crear una tremenda confusión en el mercado londinense, la cual se manifestó en un descenso de los precios. En 1866 , un buen sherry se cotizaba a unas $30 £$ por bota ${ }^{12}$.

El reajuste de los precios, que en realidad era una vuelta a la normalidad tras una covuntura extraordinaria, no fue suficiente para evitar la crisis del comercio de sherry. Por el contrario, quizá contribuyó a acentuarla al propiciar una mayor venta de vinos comunes debido a los compromisos contraídos por los exportadores con los productores de estos caldos durante los años de escasez de sherry, $y$ a su intento, por otra parte, de compensar mediante un maver volumen de ventas la pérdida de su valor. De ahí que las exportacio. nes de vinos desde Jerez, en las que el sherry tenía una proporción cada vez menor, continuara aumentando hasta 1873. A partir de esta fecha, las exportaciones de vinos de Jerez, y en particular de sherry, inician una caída de la que no se recuperarían en la pasada centuria. Fue decisiva en esta caída la vinculación casi exclusiva del comercio de sherry a un solo mercado, Gran Bretaña. La pérdida de una reputación tradicional, el cambio en el gusto de los consumidores, y quizá también la depresión finisecular en Inglaterra, dieron lugar a que una crisis covuntural del comercio del sherry, en los primeros años sesenta — crisis de escasez de los caldos-, se agravara por la competencia de otros vinos procedentes de la misma comarca y se convirtiera en el punto de partida de una crisis de mayores proporciones que afectó a toda la industria del sherry establecida en Jerez durante el último tercio del siglo xix.

Los productores jerezanos intentaron paliar la pérdida del mercado británico mediante la exportación de sherry a otros países donde ya era conocido y gozaba de cierta aceptación. Sin embargo, estos esfuerzos sólo se vieron coronados por un moderado éxitc, ya que ningún otro país llegó a sustituir a Gran Bretaña en el consumo de sherry. En los países del norte de Europa, el consumo apenas experimentó variaciones; las exportaciones se mantuvieron de forma bastante estable entre un 12,5 y un 15 por 100 en los primeros años setenta, y el volumen, en términos absolutos, no presentó mayores oscilaciones. Los Estados Unidos mantuvieron su participación sin grandes cambios, en torno a un 5 por 100 , lo que realmente indica una baja en el consumo, al descender el volumen total de las exportaciones. Fue el mercado hispanoamericano el que reaccionó de forma más positiva a la penetración del

"CR 1866, vol. LXX, p. 328.

${ }^{12}$ CR 1867-68, vol. LXVIII, p. 525. 
sherry, fenómeno interesante por cuanto su consumo era mínimo con anterioridad: un 1,5 por 100 del total exportado en $1872^{13}$. Estas cifras ponen de manifiesto la vinculación del consumo de sherry a la civilización anglosajona, Gran Bretaña y sus colonias especialmente, y explican en parte el escaso éxito que tuvieron los intentos de difusión en otras áreas.

La exportación de esparto desde los puertos del Sureste a Gran Bretaña, que se inicia en la segunda mitad del siglo $\mathrm{xIx}$, es un fenómeno relativamente desconocido, aunque no por ello poco importante, como se deduce del cuadro 1. En 1862, fecha inicial del despegue, se embarcaron $15.938 \mathrm{Tm}$, tres veces más que el año anterior y cinco veces más que el volumen medio que había salido del país desde 1850. Hasta 1862, esas pequeñas exportaciones estaban, según parece, destinadas a Italia, para usos similares a los que se daba a esta planta en España: confección de cuerdas, serones, alpargatas y similares ${ }^{14}$. A partir de esta fecha, el grueso de las exportaciones se destinó a las Islas Británicas, donde se utilizó en la fabricación de papel ${ }^{15}$.

El alza del volumen de las exportaciones tuvo un carácter especialmente marcado entre 1862 y 1869, año en que se alcanzó un máximo de $78.917 \mathrm{Tm}$, del que un 90 por 100 estuvo destinado a Gran Bretaña. En los diez años siguientes, el volumen de las exportaciones descendió hasta estabilizarse en torno a unas 30 ó $40.000 \mathrm{Tm}$ anuales.

La participación británica pasó de un 92 por 100 en el decenio 1869-1878 a un 97 por 100 a partir de 1879 , prueba evidente de la vinculación de las exportaciones de esparto a este mercado. Si tenemos en cuenta que el porcentaje de las exportaciones a las Islas Británicas aumentó al mismo tiempo que descendía el volumen total de las exportaciones, podemos afirmar que esta expansión del mercado inglés se hizo a costa de otros compradores, como el ya mencionado de Italia. El esparto español, sin embargo, representaba un porcentaje cada vez menor de las importaciones británicas. Así, pasó de ser un 75 por 100, aproximadamente, en los primeros años sesenta, a ser un 50 por 100 en los años inmediatamente posteriores a 1869 , y algo menos de un 20 por 100 a partir de $1880^{16}$.

Los años sesenta y setenta corresponden, por otra parte, a una etapa de alza en los precios del esparto, alza que culminó en 1882, cuando el esparto se cotizó a $7 £$ por tonelada. A partir de esta fecha, y en apenas unos años, los precios cayeron hasta situarse en torno a las $4 £$ en 1888 . Volvieron, pues,

" CR 1874, vol. LXVI, pp. 446-448; CR 1878, vol. LXXIII, pp. 508.516.

it CR 1864, vol. LXI, p. 344

"La utilización de esparto en la industria papelera británica es un tema que estudio más a fondo en Núñez (en prensa). 
tras unos años de bonanza, a los valores iniciales de comienzos de los sesenta ${ }^{17}$

La exportación de plomo andaluz ya era importante en las primeras décadas del siglo, cuando las provincias costeras de Almería y Granada, en especial la primera, eran las principales productoras. El período comprendido entre 1860 y 1880, aproximadamente, es interesante por los cambios que tuvieron lugar en la exportación de plomo andaluz. En estos años se inicia un fuerte descenso de la producción de plomo en Andalucía, que pasó de ser un 70 por 100 en 1861-65 a menos de un 50 por 100 en 1876-80. Esta caída se debió al progresivo decaimiento de la producción en los yacimientos costeros, de Almería en particular, que no pudo ser contrarrestado por la mayor actividad registrada en los distritos mineros del interior, en las provincias de Jaén y Córdoba, como se advierte en el cuadro 2. La explotación «artesanal» de los yacimientos costeros fue sustituida por una explotación en gran escala, a menudo con la ayuda de capital, técnicas y formas de organización extranjeras, en los distritos mineros del interior ${ }^{18}$.

La exportación de plomo español era un 90 por 100 de la producción total, lo que, suponiendo que la exportación de plomo andaluz fuera también de un 90 por 100 de la producción, nos permite sugerir que la exportación de plomo andaluz suponía entre un 5 y un 6 por 100 del valor de las exportaciones españolas. A escala regional, esto supondría, probablemente, entre un 10 y un 15 por 100 del sector exterior.

\section{CUADRO 2}

Producción andaluzo de plomo en barras. Porcentaje del total español. Medias quinquenales para el período 1861-1880

\begin{tabular}{|c|c|c|c|c|c|c|}
\hline & Años & $\stackrel{\text { (1) }}{\text { Almeria }}$ & $\stackrel{(2)}{\text { Granada }}$ & $\begin{array}{l}(3) \\
\text { Jaén }\end{array}$ & $\begin{array}{c}\text { (4) } \\
\text { Córdoba }\end{array}$ & $\begin{array}{c}\text { (5) } \\
\text { Andalucia }\end{array}$ \\
\hline $\begin{array}{l}1861-65 \ldots \\
1866-70 \ldots \\
1871-75 \ldots \\
1876-80 \ldots\end{array}$ & $\begin{array}{llllll}\ldots & \ldots & \ldots & \ldots & \ldots & \ldots \\
\cdots & \ldots & \ldots & \ldots & \ldots & \ldots \\
\cdots & \ldots & \ldots & \ldots & \ldots & \ldots \\
\cdots & \ldots & \ldots & \ldots & \ldots & \ldots \\
\cdots & \ldots & \ldots & \ldots & \ldots & \ldots\end{array}$ & $\begin{array}{l}39 \\
33,5 \\
25,1 \\
17,3\end{array}$ & $\begin{array}{l}5 \\
3,3 \\
0,6 \\
0,2\end{array}$ & $\begin{array}{l}22,7 \\
16,3 \\
28 \\
19,7\end{array}$ & $\begin{array}{l}2,9 \\
5,5 \\
4,2 \\
7,2\end{array}$ & $\begin{array}{l}69,8 \\
69,6 \\
65,8 \\
48,6\end{array}$ \\
\hline
\end{tabular}

Fuente: Nadal (1975) (apéndice sobre la producción de plomo española).

\footnotetext{
${ }^{17}$ CR 1871, vol. LXV, p. 777.

16 Véase Nadal (1972 y 1975).
} 


\section{Las exportaciones y la economía andaluza}

Se ha escrito mucho sobre la contribución del sector exterior al desarrollo económico regional, sin que hasta el momento se haya encontrado evidencia histórica suficiente como para que cualquier postura pueda ser considerada concluyente. El caso de Andalucía en el siglo xIX subraya, una vez más, la complejidad del problema y la insuficiencia de estos modelos teóricos para resolverlo. Aquí voy a discutir dos de los temas que han sido considerados más decisivos: en primer lugar, la contribución del sector exterior a la expansión del mercado interno y, por tanto, de la economía de mercado frente a la de subsistencia dentro de la región, y, en segundo lugar, su contribución al aumento de la productividad de la economía, en este caso la andaluza, empezando por aquellos sectores más estrechamente vinculados a ese sector. La ausencia de cambios profundos y decisivos en uno y otro sentido, concluiré a continuación, pudo haberse debido a una capacidad de transformación relativamente escasa de la economía andaluza y al peso real del sector exterior dentro de la misma, temas sobre los que habría que seguir estudiando.

Tanto la expansión del mercado interno como el aumento de la productividad constituyen la base de toda economía desarrollada, e implican cambios más duraderos y profundos en la estructura social y económica de la población que la mera respuesta inmediata al estímulo de la demanda externa. A su vez, ambos factores están relacionados entre sí: la aparición y consolidación de un mercado interno es rasgo fundamental de modernización económica, por cuanto lleva consigo un aumento de la productividad, a través de la redistribución de la población activa, y, por consiguiente, un aumento de la renta y de los niveles de consumo internos.

En términos generales, se admite que la aportación del sector exterior a la aparición del mercado interno varía según sea la forma en que se distribuya la renta y los beneficios del comercio. La mayor diferencia se da entre las actividades agrícolas y las mineras, debido a las peculiaridades de cada tipo de explotación, por lo que las vamos a estudiar por separado.

En la agricultura de exportación se considera que existen dos tipos fundamentales de explotación: las grandes plantaciones trabajadas por mano de obra asalariada, incluso esclava en ocasiones, y la pequeña o mediana explotación de granjeros propietarios que producen directamente para el mercado. En el primer caso, los beneficios derivados de la exportación quedan concentrados en muy pocas manos, y se supone que contribuyen a la formación de capital, que, a su vez, puede ser reinvertido en la plantación, empleado en la industria y en los servicios o dedicado a costear importaciones de lujo ${ }^{19}$. No es frecuente que contribuya, pues, directamente a la formación del mercado interno, ya que no

19 R. E. Baldwin (1964). 
hay una verdadera distribución de los beneficios entre un amplio sector de la población. En la región andaluza existen algunos sistemas de explotación agrícola, como el de los espartizales del Sureste, que se aproximan, a grandes rasgos, a este patrón.

El esparto, también conocido como atocha, es una planta de la familia de las gramíneas que crece espontáneamente en suelos calizos, casi totalmente carentes de mantillo, y en climas subáridos. De ahí que las principales zonas productoras de esparto en la Península se encuentren en el Sureste, entre las serranías litorales e interiores de las provincias de Almería y Murcia, Granada y Albacete. El esparto se utilizaba tradicionalmente en la fabricación de serones, cuerdas y objetos similares, como combustible en los boliches $v$ en los hogares y en otras pequeñas actividades, por lo que tan sólo se recogía esporádicamente y según las necesidades del momento. Hasta los años sesenta de la pasada centuria no se le puede considerar un producto de exportación, a pesar de que en ocasiones se hubieran enviado algunas toneladas a Italia. La aparición de la demanda británica de esparto para la fabricación de papel fue lo que hizo cambiar notablemente la situación ${ }^{20}$.

El aumento del volumen de atocha recogida con vistas a su exportación se debió a una mayor utilización de los montes y baldíos, donde normalmente crecían más plantas de las que se consumían localmente, y al empleo de un mayor número de jornaleros. Cabe preguntarse, pues, cómo afectó a los distintos grupos sociales de la región este súbito aumento de las exportaciones. A falta de un estudio sobre salarios y beneficios, hay algunos indicios que nos permiten suponer que el impacto de estas exportaciones sobre la capacidad adquisitiva y sobre la formación del mercado local fue pequeña. Parece ser que los nuevos salarios de los jornaleros empleados en la recogida regular de la atocha apenas llegaron a compensar el decaimiento de la industria local del esparto por escasez de materia prima ${ }^{21}$. Por otra parte, casi al mismo tiempo que se inició la exportación de esparto a Gran Bretaña, distintas corporaciones municipales, entre las que se encontraba incluso la de Almería capital, elevaron sus quejas al Gobierno solicitande la regulación de las subastas de esparto y el control de sus exportaciones ${ }^{22}$. De esto se deduce, pues, que ni los jornaleros, que encontraron un nuevo empleo estacional, ni los avuntamientos propietarics de los espartizales, que los arrendaban para su explotación, obtuvieron grandes ventajas de la exportación de esparto. Quizá fueron

${ }^{20}$ Sobre este tema, véanse los CR, en especial el Report by Mr. Consul Marks on the Atocha Plant, known in commerce as «Espartow, or Spanish Grass (Botanica Stipa), 1866, vol. LXIX, pp. 506-513.

${ }^{21}$ Los informes consulares franceses, Faits Commerciaux, señalan la crisis de estas industrias y hacen referencia a las importaciones de esparto para contrarrestar la menor oferta para el mercado interior.

${ }_{22}$ En respuesta a estas peticiones se promulgó la Real Orden de 26 de marzo de 1864. 
los comerciantes locales, quienes compraban el esparto in situ en las subastas de estas tierras municipales y organizaban su recogida y exportación, los que mayores beneficios obtuvieron de este comercio. Habría que rastrear en los ahorros e inversiones de este grupo los efectos del comercio de exportación.

El segundo tipo de explotación agraria con vistas a la exportación a que antes hice referencia, el de la pequeña o mediana propiedad en la que el propietario trabaja directamente su tierra, se supone que da lugar a una amplia distribución de la renta y de los beneficios derivados de la exportación de sus productos. Este tipo de explotación puede contribuir, pues, de una forma más directa e inmediata que el anterior, a la expansión del mercado interno, aunque se hava afirmado con frecuencia que el igualitarismo de la pequeña propiedad perjudica a la inversión y a la innovación en la agricultura ${ }^{23}$. La agricultura vinícola de Jerez es un ejemplo de este tipo de explotación en Andalucía.

La especialización de la agricultura jerezana en la producción de vinos se inició en el siglo xvin, al consolidarse la demanda británica de sherry. El consumo de sherry, como vimos, no llegó a arraigar en España como lo hizo en los países anglosajones, por lo que más del 90 por 100 de la producción se exportaba. El sherry más apreciado por su calidad era el que se producía en las albarizas, tierras blancas de las colinas no muy abundantes. Por sus cualidades, estas tierras se habían dedicado desde antiguo al cultivo de la vid en régimen de pequeñas y medianas explotaciones trabajadas directamente por su propietario. El cuidado y mantenimiento de las viñas requería, sin embargo, el empleo de trabajadores asalariados durante ciertas épocas del año. Algunas de las labores que se encargaban a estos jornaleros eran muy delicadas e importantes para la salad de los viñedos, por lo que requerían un trabajo especializado $y$, por tanto, unos salarios superiores a la media regional. Una vez realizada la vendimia, los cosecheros vendían la uva a los bodegueros, quienes se encargaban de transformarla en mosto y de almacenarla hasta que los exportadores le daban salida. Las industrias relacionadas con la producción y exportación de sherry proliferaron en Jerez y, con ellas, mejoró el nivel de vida de sus habitantes, así como su capacidad de organización, como se pondría de manifiesto en los momentos de crisis económica y social que siguieron a la caída de las exportaciones en el último tercio del siglo ${ }^{24}$.

De la organización de la producción de vinos en Jerez se deduce que hubo una cierta distribución de la renta derivada de las exportaciones entre los propietarios de viñedos, los bodegueros, los exportadores y los trabajadores asalariados que se emplearon en muchas de estas actividades. Cabría, pues,

${ }^{23}$ Véase una reciente afirmación de este principio en P. O'Brien y otros (1977).

${ }^{44}$ Véanse J. Simpson (1983, inédito), T. Kaplan (1977) y los CR de Cádiz y Sevilla. 
suponer que esto contribuyó a la expansión de la economía de mercado en la comarca de Jerez, tal y como se sugiere en el modelo teórico.

En las explotaciones mineras también existen, por su parte, los dos grandes tipos que hemos señalado en las agrícolas: la gran explotación, con características de empresa moderna, tales como fuertes inversiones de capital, aportaciones de tecnología y empresariado competente, y, por otra parte, la pequeña explotación, casi familiar, con métodos tradicionales y bajos rendimientos. A diferencia de la agricultura, sin embargo, en la minería era un requisito indispensable para su modernización la concentración de las explotaciones. De ahí que en la segunda mitad del siglo xIx apenas sobrevivieran casos aislados de pequeñas explotaciones mineras, mientras se afirmaban las grandes empresas. La contribución de éstas al crecimiento económico de la región podía venir, de un lado, de la acumulación de beneficios o formación de capital $\mathrm{y}$, por tanto, de las inversiones posteriores en esta misma actividad extractiva o en otras y, de otro, de la creación de empleo con salarios relativamente elevados. La nacionalidad de las empresas mineras es, pues, aspecto fundamental y decisivo, ya que, normalmente, aquellas de crigen extranjero tendían a reexportar los beneficios obtenidos de la exportación de mineral ${ }^{25}$.

Durante la segunda mitad del siglo xix, y en los principales distritos mineros de Andalucía, predominaron las compañías extranjeras, sobre todo británicas, lo que, sin duda, supuso una serie de trabas a los posibles efectos de arrastre que la exportación del mineral hubiera podido tener. Este predominio vino determinado por las dificultades que planteaba la explotación de algunos de estos yacimientos, cuya solución requería unas inversiones de capital y tecnología que no estaban disponibles en la región, y se vio facilitado por la progresiva liberación de la legislación minera española a partir de 1859 , y en especial de la Ley de Bases de 1868, y por la mejora de las comunicaciones con la construcción de diversas líneas ferroviarias. La explotación del plomo de Linares y de las piritas ferrocupríferas de Huelva ilustran esta presencia extranjera en la minería andaluza.

El proceso de transformación y expansión de la minería en el distrito de Linares se inició, en 1849, con el arrendamiento de la mina del Pozo Ancho por una compañía británica, la Linares Lead ${ }^{26}$. En 1866, los ingleses controlaban ya seis minas - las de Pozo Ancho, La Fortuna, Alamillos, La Tortilla, San Roque y Collado del Lobo- y un 55 por 100 del capital invertido en la minería en Linares. Entre estas seis minas disponían de 16 máquinas de vapor, de un total de 28 en el distrito, y empleaban 1.800 hombres, de 3.100 dedicados a la minería ${ }^{27}$. En 1880 eran ocho las compañías de capital inglés:

${ }^{25}$ Véase J. V. Levin (1960), en especial su discusión sobre el comportamiento de los factores externos en la exportación del guano chileno.

${ }_{26}$ Véase J. Nadal (1975), capítulo 3.

27 CR 1867-68, vol. LXVIII, p. 675. 
tres de ellas habían sobrevivido desde 1866 - Pozo Ancho, La Fortuna y Alamillos - y otras cinco se habían creado durante estos años. Las compañías británicas seguían siendo las que empleaban mayor capital, número de máquinas de vapor y de mineros de todo el distrito ${ }^{28}$. Junto a ellas existían en Linares compañías francesas, belgas y españolas. Estas últimas aumentaron más rápidamente en número a partir de finales de los años ochenta, ante la retirada progresiva de otras compañías extranjeras con la caída de los precios del plomo en el mercado de Londres. La concentración de la actividad minera linarense en manos extranjeras «se extremaba en el ramo del beneficio", siendo el predominio británico en este sector muy acusado. En 1880 existían en Linares cinco fundiciones de plomo, tres de ellas trabajando a pleno rendimiento y dos sólo parcialmente. De las tres primeras, dos pertenecían a compañías británicas ${ }^{29}$. Apenas diez años más tarde, las cinco fundiciones habían quedado reducidas a tres - una inglesa, una francesa y una española-. De ellas, la británica era la que beneficiaba un mayor volumen de mineral de plomo, el 56 por 100 de plomo en barras de este distrito ${ }^{30}$.

En Huelva, el predominio extranjero, cabe decir británico, en la explotación de las piritas ferrocupríferas era aún más completo. Diversas compañías alemanas, francesas e inglesas se sucedieron en la primera mitad de siglo con escaso éxito, hasta que, finalmente, se crearon dos grandes compañías, The Rio Tinto Co., con domicilio en Londres, y The Tharsis Sulphur and Copper Co., con domicilio en Glasgow, que dieron el impulso definitivo a la minería onubense en la década de 1870. Al amparo de estas dos proliferaron otras compañías menores, entre las que destacaron siempre las británicas y alguna que otra francesa ${ }^{31}$. La principal razón de este predominio británico estuvo en los dos problemas más graves que se oponían a una eficaz explotación: el transporte del mineral hasta la costa para su exportación y el tratamiento de los minerales que permitiera utilizar su elevado contenido de azufre. Tan sólo los británicos reunían el capital y la técnica necesarios para hacer frente a ambos problemas, al mismo tiempo que contaban con el principal mercado para el azufre de las piritas: la industria química británica.

El predominio de compañías extranjeras en las explotaciones mineras andaluzas, según se desprende de los ejemplos señalados de Linares y Huelva, permite, pues, suponer que una elevada proporción de los beneficios derivados de la exportación del mineral permanecieron en el extranjero, con la excepción de la renta pagada al Estado o a los concesionarios anteriores por el arrendamiento o compra de las minas, y los salarios de los mineros. Este últi-

28 CR 1882, vol. LXXI, p. 564

29 CR ibid., p. 565.

${ }^{30}$ CR 1890, vol. LXXVII, p. 87.

"CR 1878, vol. LXXIII, p. 478. 
mo es el aspecto que nos interesa destacar aqui, por su mayor impacto sobre la economía regional ${ }^{32}$.

Como los jornaleros agrícolas especializados de la campiña jerezana, también los mineros disfrutaban de unos salarios superiores a la media regional. Esto contribuyó, sin duda, a que los pueblos mineros se convirtieran en focos de atracción de la población. Linares, por ejemplo, pasó de tener 6.000 habitantes en 1849 a más de 36.000 en 1877 , es decir, multiplicó su población por seis en apenas un cuarto de siglo. En Huelva, tan sólo la compañía de Río Tinto llegó a emplear 11.260 hombres en 1894. Ahora bien, los posibles efectos multiplicadores de estos núcleos de niveles de renta superiores se vieron limitados por la estrecha vinculación que, frecuentemente, se daba entre los mineros y las compañías para las que trabajaban, a través de las cooperativas de la misma empresa, donde los mineros podían ser obligados a efectuar sus compras ${ }^{33}$.

A modo de conclusión, se puede decir que el sector exterior andaluz dio lugar en ocasiones a un aumento de los salarios relativos, en especial en la agricultura de pequeña y mediana propiedad y en la minería, cuya contribución a la formación del mercado interno no parece, sin embargo, haber sido considerable. A falta de un estudio detenido sobre los salarios andaluces durante el siglo XIX, cabe señalar que las diferencias entre los sectores más dinámicos y los más atrasados no tenía que ser muy grande, dado que se trataba de una economía con oferta ilimitada de mano de obra ${ }^{34}$.

La existencia de una oferta ilimitada de mano de obra, con la consiguiente presión a la baja sobre los salarios, y la presencia de intereses y empresas extranjeras en las actividades que propiciaban en mayor medida la acumulación de capital, con la consiguiente reexportación de los beneficios, fueron dos de los principales obstáculos que limitaron la expansión del mercado interno a partir del comercio de exportación. Tampoco contribuyó a la consolidación del mercado interno el mal estado de las comunicaciones interregionales $\mathrm{y}$, por tanto, el aislamiento en el que prosperaron las distintas actividades exportadoras.

Durante la segunda mitad del siglo xix mejoraron notablemente las comunicaciones con la construcción de diversas líneas de ferrocarril. Su vinculación al comercio de exportación queda claramente de manifiesto mediante una ojeada al mapa de los ferrocarriles andaluces, y en especial al trayecto de las líneas

32 Para España, véanse los trabajos de Broder (1976), y para un caso andaluz, la excelente obra de Ch. E. Harvey (1981), en especial el capítulo 4, que hace referencia a la distribución de los beneficios de la explotación y mantiene, contra otras tesis, que el Estado español obtuvo un precio justo por el enajenamiento de los yacimientos de Río Tinto en condiciones de competencia abierta en el momento de la adjudicación.

${ }_{33}$ J. Franco Quirós (1979), p. 509, y Ch. E. Harvey (1981), p. 124.

${ }^{34}$ W. A. Lewis (1963). 
principales: Madrid-Sevilla-Cádiz y Madrid-Córdoba-Granada. La primera atraviesa la región de Noreste a Suroeste, abriendo la comunicación entre las comarcas más ricas y fértiles del interior y el Atlántico, en el puerto de Cádiz. La segunda corta a la primera casi perpendicularmente de Norte a Sur, poniendo en contacto el interior con el Mediterráneo, en Málaga. La vinculación del ferrocarril a la exportación queda aún más clara en tramos concretos como el de Jerez-Puerto de Santa María, uno de los primeros del país, y el de ciertos ferrocarriles denominados mineros, como los de Río Tinto, Linares-Almería, etcétera. La contribución de estas líneas a la integración del mercado interno andaluz no parece, sin embargo, haber sido tan importante como su contribución a la expansión del comercio exterior.

El segundo tema que se plantea en esta sección es la aportación del sector exterior, y más concretamente la especialización de la producción andaluza con exportadores como en los internos. Esta cuestión queda por ahora a un nivel más hipotético, si cabe, que el anterior, dado el estado actual de la investigación. El aumento de la productividad puede ser una respuesta momentánea al estímulo de la demanda externa. Normalmente, pues, sería consecuencia de un mayor empleo de la población activa, entre la que el paro, tanto real como encubierto, era una constante muy elevada, $y$, por otra parte, de la puesta en explotación de recursos naturales antes abandonados o aprovechados por debajo de sus posibilidades. La amenaza más inmediata que se cierne sobre este aumento de la productividad es el agotamiento temporal o la extinción de los recursos, debido en ocasiones al mal uso -como ciertamente ocurrió con el esparto-y en ocasiones a factores naturales - como frecuentemente ocurre en la minería - Para que el aumento de la productividad sea duradero es necesario que la región aproveche no sólo las oportunidades más inmediatas que le ofrece el mercado exterior, sino las posibilidades de introducir mejoras técnicas rentables a más largo plazo. Esta posibilidad, según Myint, es mayor en las grandes explotaciones agrícolas que en las pequeñas o medianas, y sobre todo en la minería, debido a una mayor concentración de los beneficios que permite sufragar las inversiones. Aún más importante que estos efectos, en opinión del mismo autor, es el papel que estas actividades más dinámicas desempeñan como agentes difusores de tecnología y educación ${ }^{35}$. Este es el aspecto de la minería andaluza que vamos a comentar brevemente.

La evolución de la minería en Linares es un caso típico de difusión de tecnología en la región exportadora. Cuando a comienzos de la década de los años cincuenta aparecieron las primeras compañías extranjeras en la provincia de Jaén, la explotación del plomo linarense estaba casi totalmente paralizada debido a las dificultades que planteaba el desagüe en pozos cada vez más profundos. La solución a este y otros problemas hacía necesaria una

${ }^{35}$ H. Myint (1973), capitulo 4. 
fuerte inversión en técnicos y equipo que no existían en la Península, y a cuyo coste sólo ciertas compañías podían hacer frente. Los concesionarios españoles, que normalmente trabajaban a una escala muy limitada, no estaban en condiciones de introducir estas mejoras, por lo que la tarea correspondió a las compañías extranjeras, que finalmente se interesaron en este distrito. Al amparo de éstas pudieron proliferar nuevas compañías españolas, que llegaron a superar a las extranjeras en número, aunque no en volumen de producción. Su éxito se debía a que siguieron el sistema de explotación introducido por las compañías británicas, y también a que emplearon técnicos y maquinaria procedentes de Gran Bretaña ${ }^{36}$. En la década de los años ochenta, cuando las empresas extranjeras iniciaron su retirada de Linares, las compañías españolas ya estaban en condiciones de sustituirlas y de mantener viva la minería en esa región. Es decir, que gracias a la previa difusión de tecnología, los españoles pudieron proseguir con la explotación del plomo en las sierras del interior.

En la explotación de las piritas ferrocupríferas de la provincia de Huelva no se produjo, sin embargo, un fenómeno de difusión tecnológica comparable al de Linares. La escala de estas explotaciones quedaba fuera de las posibilidades de los empresarios andaluces. Ahora bien, contribuyó eficazmente a elevar el nivel de educación en los pueblos mineros que crecieron al amparo de las nuevas compañías, y a la formación de capataces y obreros especializados cuyo empleo se disputaban en todos los distritos mineros del país ${ }^{37}$.

El sector exterior contribuyó, pues, de alguna forma a la formación de capital humano: hizo disminuir el analfabetismo entre los trabajadores y mejorar su preparación técnica - Río Tinto-, facilitó la adopción de nuevos métodos de producción y organización de las empresas - Linares y Jerez- y fomentó la transmisión de tecnología extranjera - Linares-. Esto nos permite suponer que la producción aumentó en algunas de las actividades más estrechamente vinculadas al comercio exterior.

\section{Conclusiones}

Podría aventurarse que el comercio de exportación andaluz tuvo un menor impacto sobre la economía andaluza del que habría cabido esperar. Su contribución a la expansión del mercado interno se vio limitada, en primer lugar, por la oferta ilimitada de mano de obra y su presión a la baja sobre los salarios, lo que minimizó las diferencias salariales entre estos sectores más dinámicos y los de subsistencia, $y$, en segundo lugar, por el pequeño tamaño

${ }^{36}$ Véanse los CR mencionados anteriormente al estudiar la minería en Linares.

${ }^{37}$ Ch. E. Harvey (1981), p. 124. 
de estos núcleos exportadores en relación con la economía andaluza en su conjunto, así como su relativo aislamiento o falta de conexión entre ellos. Por otra parte, su aportación a la formación de capital se vio reducida por la existencia de intereses extranjeros en aquellos sectores de exportación que la favorecieron. Finalmente, su contribución a la mejora de la productividad no parece haber sido inmediata, sino más bien indirecta, a través de una cierta transmisión de tecnología, de modos de organización empresarial y de educación en general, y muy dispersa.

¿A qué se debió, pues, lo que parece ser un impacto tan limitado del sector exterior andaluz sobre la economía de la región? Entre las diversas razones que se han aducido, en otros casos parecidos, destacan las siguientes: la falta de capacidad de transformación, es decir, la escasa flexibilidad para pasar de una actividad económica a otra, en este caso concreto para sustituir unos productos de exportación por otros, o unos mercados por otros, o para pasar a otras actividades no directamente vinculadas con el comercio de exportación. La dificultad de cambio se ha achacado al peso de las instituciones, que actúan como lastre frente a los intentos de renovación, y a la escasez de capital humano, lo que impide que se adviertan muchas de estas posibilidades de cambio. También se ha dicho que el impacto del sector exterior sobre la economía regional depende, en gran medida, del tamaño del sector y de su ritmo de crecimiento, aunque no se ha podido definir cuál habría de ser uno y otro ${ }^{38}$.

Para entender el caso de Andalucía durante el siglo xix parece que habría que introducir una nueva variante a esta última explicación. El sector exterior andaluz, aparentemente importante dentro del total español, así como muy rico y diversificado, puede encubrir un problema de economías monoexportadoras que comparten mercados similares, pero carecen de cualquier otro tipo de conexión entre ellas. Esta falta de comunicación entre los distintos núcleos exportadores dificultaría, lógicamente, los efectos multiplicadores del sector exterior en su conjunto, y explicaría la falta de una mayor capacidad de transformación. Ahora bien, si admitimos la hipótesis de la compartimentación y aislamiento de las exportaciones andaluzas, habría que preguntarse también si la desconexión del sector exterior no refleja la falta de integración económica a nivel regional. Mi hipóttsis, pues, para entender el limitado impacto del sector exterior sobre la economía de Andalucía durante el siglo xix es que la región aún no había alcanzado un mínimo grado de integración económica. Para comprobar esta hipótesis, propongo realizar un estudio comparado del mercado interno en distintas regiones españolas, en especial aquellas que también tuvieron un sector exterior importante, como

3* N. Leff (1973). 
Cataluña, el País Vasco y el País Valenciano. Este estudio nos permitiria apreciar hasta qué punto influyó el nivel de integración del mercado interno en el alcance del impacto del sector exterior.

\section{BIBLIOGRAFIA}

Andrés Alvarez, V. (1943): «Historia y crítica de los valores de nuestra balanza de co. mercio", en Moneda y Crédito, núm. 4.

- (1945): "Las balanzas. Estadísticas de nuestro comercio exterior", en Revista de Eco. nomia Politica, vol. I.

Avery, D. (1974): Not on Queen Victoria's Birthday. The Story of the Rio Tinto Mines, Londres.

Baldowin, R. (1964): Economic Development and Export Growth, California.

BEREND, 1. T., y RANKI, G. (1980): "Foreign Trade and the Industrialization of the European Periphery in the 19th Centurym, en Journal of European Economic History. vol. 9 , núm. 3 (invierno), pp. 539-584.

Bernal, A. M. (1974): La propiedad de la tierra y las luchas agrarias andaluzas, Barcelona.

- (1979): «El latifundio y su evolución», en ARTol.A, M., et al.; El latifundio. Propie. dad y explotación, siglos XVIII y XIX.

Bernal., A. M., y Drain, M. (1975): Le's campagnes sevillanes aux XIX-XX siècles, París, Publicaciones de la Casa de Velázquez.

Broder, A. (1976): «Les investissements étrangers en Espagne au xix siècle: Méthodologie et quantificationm, en Revue d'Histoire Economique et Sociale, vol. 54, núm. 1, pp. 29-63.

Castejón Montijano, R. (1977): Génesis y desarrollo de una sociedad mercantil e industrial en Andalucia: La Casa Carbonell de Córdoba (1866-1918), Córdoba.

- (1979): «La Banca de Pedro López, de Córdoba, durante la crisis de 1866", en Actas del I Congreso de Historia de Andalucía, Córdoba.

Cin eckland, J. G. (1967): The Mines of Tharsis. Roman, French and British Enterprise in Spain, Londres.

Cruz, J. (1980): Propiedad y uso de la tierra en la Baja Andalucia: Carmona, siglos XVIII $X I X$, Madrid.

Consular Reports (CR). Informes comerciales de los cónsules británicos, 1857-1913.

Estadística General del Comercio Exterior de España, Dirección General de Aduanas, años 1850-90.

Franco Quirós, J. (1979): “Una nueva ciudad andaluza: Linares 1875m, en Actas I Con. greso de Historia de Andalucia, Córdoba.

García Montoro, C. (1978): Máluga en los comienzos de la industrialización: Manuel Agustin Heredia, Universidad de Córdoba, Instituto de Historia de Andalucía.

GonzÁlez Gordon, J. M. (1948): Jerez, Xeres, Sherry, Jerez de la Frontera.

IIArvey, Charles E. (1981): The Rio Tinto Company. An Economic History of a Leading International Mining Concern, 1873-1954.

JIMÉNEZ QUINTERo, V. A. (1979): «Mentalidad empresarial y acumulación de capital de la casa Larios de Málaga», en Cuadernos de Ciencias Económicas y Empresariales, núm. 4.

Kaplan, T. (1977): Anarchists of Andalusia 1868-1903, New Jersey.

KindLeBerger, Ch. P. (1968): «Foreign Trade and Economic Growth: Lessons from Britain and France, 1850-1913m, en FALCuS, M. E.: Readings in the History of Economic Growth.

Kravis, I. B. (1970): «Trade as a Handmaiden of Growth: Similarities between the Nineteenth and the Twentieth Centuries", en Economic Journal, vol. LXXX, pp. 850-872. 
LefF, Nathaniel H. (1973): «Tropical Trade and Development in the Nineteenth Century: The Brazilian Experience», en Journal of Political Economy, vol. 81 (mayo), pp. 678-696.

Levin, I. V (1960): The Export Economies: Their Pattern of Development in Historical Perspective.

LEWIS, W. A. (1963): “El desarrollo económico con oferta ilimitada de trabajo", en Agarwala, A. N., y Singh, S. P.: La economía del subdesarrollo, Madrid, Tecnos.

Morilla Critz, José (1978): Gran capital y estancamiento económico en Andalucia. Banca y terrocarril en Málaga en el siglo XIX. Universidad de Córdoba, Instituto de Historia de Andalucía.

- (1984): «El ferrocarril de Córdoba a la cuenca hullera de Espiel y Bélmez, 1852-1880", en Revisla de Historia Económica. año II, núm. 1, pp. 83-104.

Muñoz Dueñas, M. D. (1979): «Importancia del factor tecnológico en el crecimiento económico del distrito de Linares 1850-1881", en Actrs I Congreso de Historia de Andalucia, vol. I, Córdoba.

Myint, H. (1973): The Economics of the Developing Countries, Londres, 4." ed. revisada.

NADAL, J. (1972): “Industrialización y desindustrialización en el Sureste español, 1817. 1913\%, en Moneda y Crédito, núm. 120, pp. 3-76.

- (1975): El fracaso de la Revolución Industrial en España, Barcelona.

Núñez, Clara Eugenia (en prensa): El comercio exterior y los problemas del desarrollo económico en Andalucia, 1850-1890, Sevilla.

Nurkse, R. (1961): Patterns of Trade and Development, New York.

O'Brien, P. K.; Hesth, D., y Keyner, C. (1977): "Agricultural Efficiency in Britain and France, 1815-1914», en lournal of European Economic History, vol. 6, núm. 2.

Prados de la Escosura, Leandro (1981): «Las estadísticas españolas de comercio exterior, 1850-1913. El problema de las valoraciones», en Moneda y Crédito, núm. 156 (marzo), pp. 43-60.

- (a) (1982): Comercio exterior y crecimiento económico en España, 1826-1913: Tendencias a largo plazo, Banco de España, Servicio de Estudios, Estudios de Historia Económica, núm. 7 .

- (b) (1982): "Comercio exterior y cambio económico en España (1792-1849)», en FoNtana, J. (ed.): La economía española de al final del Antiguo Régimen, vol. III: Comercio y colonias, Madrid.

- (1983): «El sector exterior en la economía andaluza durante el siglo XIX» (conferencia inédita pronunciada en Sevilla).

Prados de la Escosura, L., y Tortella Casares, G. (1983): «Tendencias a largo plazo del comercio exterior españolm, en Revista de Historia Económica, año I, núm. 2

Quirós Linares, F. (1962): "El comercio de los vinos de Jerezm, en Estudios Geográficos, tomo XXIII, núm. 86, pp. 29-44.

SÁNCHEZ-AlboRNOZ, N. (1970): "Cádiz, capital revolucionaria, en la encrucijada económica", en Lida, C., y Zavala, I.: La Revolución de 1868.

- (1968): "Los bancos y las sociedades de crédito en provincias: 1856-1868», en Moneda y Crédito, núm. 104.

- (1977): España bace un siglo: una economía dual, Madrid.

SimpSON, James (inédito): «Wine Production in Jerez de la Frontera, 1850-1900m.

SPYCER, D. A. (1907): The Paper Trade, Londres.

SH ORTER, A. H. (1971): Paper-Making in the British Isles

TEDde dE LoRCA, P. (1978): «El proceso de formáblica Española, núm. 55. rriles Andaluces (1874-1880)w, en Hacieniles Andaluces (1878-1920): una empresa de

- (1980): "La Compañía de los la Restauración», en Investigaciones Económicas, marzotransporte en la España de la Restauraci

- (1981): «Burguesía, banca y mercado (1840-1874)m, en Historia de Andalucia, vol. VII

Titos Martínez, M. (1978): Crédito y aborro en Granada en el siglo XIX, Banco de Granada, 2 vols. 
- (1980): Bancos y banqueros en la bistoriografia andaluza, Instituto de Desarrollo Regional, Sevilla.

Titos Martínez, M.; Castejón Montijano, R.; Jiménez Quintero, J. A., y Garcia Montoro, G. (1980): «Empresas y empresarios andaluces en el siglo xix: una primera aproximación», en Cuadernos de Ciencias Económicas y Empresariales, núm. 6.

Tortella Casares, G. (1973): Los origenes del capitalismo en España, Madrid.

Tortella Casares, G.; Martín Aceña, P.; Sanz, J., y Zapata, S. (1978): "Las balanzas del comercio exterior español: un experimento histórico estadístico, 1875.1913m, en García Delgado, J. L., y Segura, S.: Ciencia social y análisis económico. Estudios en homenaje al profesor $V$. Andrés Alvarez, Madrid, pp. 487-513. 